\title{
Acute Kidney Injury: Lessons from Pericardiectomy
}

\author{
Jing-bin Huang, MD, * Zhao-ke Wen, MD, * Chang-chao Lu, MD, Wei-jun Lu, Xian-ming Tang, MD, \\ Xiang-wei Li, MD, Xin Deng, MD \\ Department of Cardiothoracic Surgery, The People's Hospital of Guangxi Zhuang Autonomous Region, 6 Taoyuan Road, Nanning, \\ Guangxi, China
}

\section{ABSTRACT}

Background: Acute kidney (renal) injury (AKI) is a severe and common complication that occurs in $\sim 40 \%$ of patients undergoing cardiac surgery. AKI has been associated with increased mortality and worse prognosis. This prospective study was conducted to determine the risk factors for AKI after pericardiectomy and decrease the operative risk of mortality and morbidity.

Methods: This was a prospective, observational cohort study of patients with constrictive pericarditis undergoing pericardiectomy. All patients underwent pericardiectomy via median sternotomy. Serum creatinine was used as the diagnostic standard of AKI according to Kidney Disease Improving Global Outcomes classification. All survivors were monitored to the end date of the study.

Results: Consecutive patients $(\mathrm{N}=92)$ undergoing pericardiectomy were divided into 2 groups: with AKI ( $\mathrm{n}$ $=25)$ and without AKI $(n=67)$. The incidence of postoperative AKI was $27.2 \%(25 / 92)$. Hemodialysis was required for 10 patients $(40 \%)$, and there were 5 operative deaths. Mortality, intubation time, time in intensive care unit, fresh-frozen plasma, and packed red cells of the group with AKI were significantly higher than those of the group without AKI. Both univariate and multivariate analyses showed that statistically significant independent predictors of AKI include intubation time, chest drainage, fresh-frozen plasma, and packed red cells. The latest follow-up data showed that 85 survivors were New York Heart Association class I (97.7\%) and 2 were class II $(2.3 \%)$.

Conclusions: AKI after pericardiectomy is a serious complication and contributes to significantly increased morbidity and mortality. Prevention of AKI development after cardiac surgery and optimization of pre-, peri-, and postoperative factors that can reduce AKI, therefore, contribute to a better postoperative outcome and leads to lower rates of AKI, morbidity, and mortality.

Received April 6, 2021; received in revised form April 23, 2021; accepted May 2, 2021.

*Fing-bin Huang, $M D$, and Zhao-ke Wen, $M D$, contributed equally to this work.

Correspondence: Fing-bin Huang, MD, Department of Cardiotboracic Surgery, The People's Hospital of Guangxi Zhuang Autonomous Region, 6 Taoyuan Road, Nanning, 530021, Guangxi, China; 86-771-2188205; fax: 86-7712188214 (e-mail: hjb010222@163.com).

\section{INTRODUCTION}

Acute kidney (renal) injury (AKI) is a severe and common complication that occurs in $~ 40 \%$ of patients undergoing cardiac surgery. AKI has been associated with increased mortality, worse prognosis, more days of mechanical ventilation, a higher inotropic requirement, and a longer length of stay [Meersch 2017; Calderon-Rojas 2020; Leballo 2020]. We hypothesized that improvement in prevention and treatment of AKI after pericardiectomy can decrease operative mortality and morbidity.

This prospective study was conducted to determine the risk factors for AKI after pericardiectomy and decrease the operative risk of mortality and morbidity.

\section{METHODS}

Design

This was a prospective, observational cohort study of patients with constrictive pericarditis undergoing pericardiectomy from January 2009 to June 2020 at our hospital. The most frequently carried out classifications of AKI by researchers are Risk, Injury, Failure, Loss of Kidney Function and End-Stage Kidney Disease (RIFLE), Acute Kidney Injury Network (AKIN), and Kidney Disease Improving Global Outcomes (KDIGO). RIFLE and AKIN use criteria such as change in serum creatinine level, increase of $\geq 1.5$ times from the baseline, and urine output of $<0.5 \mathrm{~mL} / \mathrm{kg} / \mathrm{h}$ for at least 6 hours [Jacob 2019]. KDIGO is based on the combination of RIFLE and AKIN criteria and has become a novel consensus classification for diagnosis of AKI; it has also been shown to have greater sensitivity in detection of AKI postoperatively than other classifications. Currently, AKI is diagnosed based mainly on sharp rises in serum creatinine levels [Howitt 2018; Jacob 2019; Sutherland 2020].

In this study, serum creatinine was used as the diagnostic standard of AKI. According to KDIGO classification, if serum creatinine increases by $\geq 0.3 \mathrm{mg} / \mathrm{dL}(26.5 \mu \mathrm{mol} / \mathrm{L})$ within 48 hours, serum creatinine is $50 \%$ higher than baseline within the first 7 days, or urine output is $<0.5 \mathrm{~mL} / \mathrm{kg} / \mathrm{h}$ for 6 hours, the patient is considered to have AKI [Howitt 2018; Jacob 2019; Sutherland 2020]. The patients were divided into 2 groups: with AKI and without AKI.

\section{Patients}

Eligibility. All patients with constrictive pericarditis undergoing pericardiectomy from January 2009 to 
Table 1. Preoperative data between the patients with and without AKI $(n=92)$

\begin{tabular}{lccc}
\hline Variable & $\begin{array}{c}\text { With AKI } \\
(\mathrm{n}=25)\end{array}$ & $\begin{array}{c}\text { Without AKI } \\
(\mathrm{n}=67)\end{array}$ & $P$ \\
\hline Male sex & $14(56)$ & $45(67.2)$ & .321 \\
Age $(\mathrm{y})$ & $53.6 \pm 2.9$ & $52.5 \pm 1.9$ & .752 \\
Weight before diuresis $(\mathrm{kg})$ & $53.8 \pm 1.6$ & $56.8 \pm 1.1$ & .170 \\
Weight2 $(\mathrm{kg})$ & $51.4 \pm 1.3$ & $53.6 \pm 0.9$ & .182 \\
Preoperative CVP $(\mathrm{mmHg})$ & $21.7 \pm 0.9$ & $20.0 \pm 0.6$ & .123 \\
Preoperative LVEDD $(\mathrm{mm})$ & $41.0 \pm 0.5$ & $40.8 \pm 0.5$ & .779 \\
Preoperative LVEF $(\%)$ & $59 \pm 1.0$ & $63 \pm 1.0$ & .007 \\
Baseline serum creatinine $(\mu \mathrm{mol} / \mathrm{L})$ & $77.2 \pm 3.7$ & $80.3 \pm 4.7$ & .695 \\
\hline
\end{tabular}

Data are $\mathrm{n}(\%)$ or mean \pm standard deviation.

Abbreviations: AKI, acute renal injury; CVP, central venous pressure; LVEDD, left ventricular end diastolic dimension; LVEF, left ventricular ejection fraction.

June 2020 at our hospital were included in the study. The diagnosis of constrictive pericarditis was confirmed by clinical presentation, echocardiographic study, chest computed tomography (CT) scan, and cardiac catheterization, as needed.

Histopathologic studies of pericardium tissue from every patient was done. The diagnosis of tuberculosis was confirmed on the basis of clinical findings and histopathologic features, including the presence of typical granuloma and caseous necrosis, and acid-fast bacilli in Ziel-Nelson tissue staining. Patients who died within the first 24 hours after surgery were excluded from the study.

Variables Investigated. Variables were evaluated including sex, age, weight, baseline serum creatinine, the need for transfusion of blood products, postoperative serum creatinine, fluid balance on operation day and postoperative days 1 and 2, the need for hemodialysis, the length of intensive care unit (ICU) stay, central venous pressure, left ventricular end diastolic dimension, left ventricular ejection fraction, chest drainage, serum creatinine after surgery, fresh-frozen plasma, packed red cells, multiple organ failure, and death.

\section{Surgical Technique}

All patients underwent pericardiectomy via median sternotomy. In patients approached via sternotomy, total pericardiectomy was performed between the 2 phrenic nerves and from the great vessels to the basal aspect of the heart. In cases of high risk of coronary artery or myocardial damage or severe bleeding, the pericardium over the right atrium or superior and inferior venae cavae was left intact. The primary intention was pericardiectomy without cardiopulmonary bypass (CPB). Postoperative death was defined as death occurring within 30 days of surgery or within the hospitalization period for the operation.
Table 2. Operative Data

\begin{tabular}{|c|c|c|c|}
\hline Variable & $\begin{array}{l}\text { With AKI } \\
(n=25)\end{array}$ & $\begin{array}{l}\text { Without AKI } \\
\qquad(n=67)\end{array}$ & $P$ \\
\hline Operative death & $5(20)$ & 0 & .000 \\
\hline Multiple organ failure & $8(32)$ & 0 & .000 \\
\hline Intubation time $(\mathrm{h})$ & $133.7 \pm 23.5$ & $54.3 \pm 6.5$ & .000 \\
\hline ICU retention time $(\mathrm{d})$ & $7.6 \pm 1.3$ & $4.5 \pm 0.5$ & .007 \\
\hline $\begin{array}{l}\text { Hospitalized time } \\
\text { postoperative (d) }\end{array}$ & $17.0 \pm 1.8$ & $15.7 \pm 1.4$ & .621 \\
\hline Postoperative CVP (mmHg) & $11.4 \pm 0.5$ & $11.7 \pm 0.4$ & .722 \\
\hline Day 0 fluid balance $(\mathrm{mL})$ & $-980.4 \pm 184.5$ & $-1377.3 \pm 96.0$ & .043 \\
\hline Day 1 fluid balance $(\mathrm{mL})$ & $83.0 \pm 543.3$ & $-882.4 \pm 119.9$ & .015 \\
\hline Day 2 fluid balance $(\mathrm{mL})$ & $-1231.3 \pm 183.3$ & $-609.7 \pm 64.6$ & .000 \\
\hline Chest drainage $(\mathrm{mL})$ & $1242.4 \pm 110.5$ & $817.5 \pm 61.9$ & .001 \\
\hline Postoperative LVEDD (mm) & $42.1 \pm 0.8$ & $42.9 \pm 0.4$ & .322 \\
\hline Postoperative LVEF (mm) & $0.65 \pm 0.01$ & $0.67 \pm 0.01$ & .229 \\
\hline $\begin{array}{l}\text { Serum creatinine, } 24 \mathrm{~h} \text { after } \\
\text { surgery }(\mu \mathrm{mol} / \mathrm{L})\end{array}$ & $99.3 \pm 4.2$ & $70.9 \pm 2.6$ & .000 \\
\hline $\begin{array}{l}\text { Serum creatinine } 48 \mathrm{~h} \text { after } \\
\text { surgery }(\mu \mathrm{mol} / \mathrm{L})\end{array}$ & $131.6 \pm 5.8$ & $70.9 \pm 3.3$ & .000 \\
\hline Fresh-frozen plasma (mL) & $1412.8 \pm 209.8$ & $317.8 \pm 55.8$ & .000 \\
\hline Packed red cells (units) & $0.58 \pm 0.2$ & $0.05 \pm 0.03$ & .000 \\
\hline
\end{tabular}

Data are $\mathrm{n}(\%)$ or mean \pm standard deviation. Day $0=$ day of surgery. Abbreviations: AKI, acute renal injury; CVP, central venous pressure; LVEDD, left ventricular end diastolic dimension; LVEF, left ventricular ejection fraction.

\section{Follow-Up}

All survivors were monitored to the end date of the study. All patients were investigated with $\mathrm{x}$-ray chest film and echocardiography once every 3 to 12 months. The patients were contacted by telephone or message or interviewed directly at the outpatient department at the last follow-up.

\section{Statistical Analysis}

Statistical analysis was performed using IBM SPSS Statistics version 24.0. Continuous variables were expressed as the mean with standard deviation and categorical variables as percentages. Paired t tests were used to make comparisons of preoperative and postoperative continuous variables. We used the Kaplan-Meier method to estimate survival rates. The $\chi^{2}$ test, Kruskal-Wallis test, or Wilcoxon rank-sum test, as appropriate, were used to evaluate relationships between the preoperative variables and selected intraoperative and postoperative variables. $P$ values $<.05$ were considered statistically significant.

\section{Ethical Aspects}

The experiment protocol for involving humans was in accordance with national guidelines and was approved by 
Table 3. Analysis of Risk Factors of Acute Kidney Injury

\begin{tabular}{|c|c|c|c|}
\hline \multirow[b]{2}{*}{ Model } & \multicolumn{3}{|c|}{ 95\% Confidence } \\
\hline & Odds Ratio & Interval & $P$ \\
\hline \multicolumn{4}{|l|}{ Univariate analysis } \\
\hline Intubation time & 1.011 & 1.005 to 1.018 & .000 \\
\hline ICU retention time & 1.125 & 1.021 to 1.239 & .017 \\
\hline Day 0 fluid balance & 1.001 & 1.000 to 1.001 & .048 \\
\hline Day 2 fluid balance & 0.999 & 0.998 to 0.999 & .001 \\
\hline Chest drainage & 1.001 & 1.001 to 1.002 & .002 \\
\hline Operative death & 30.483 & 1.370 to 678.131 & .031 \\
\hline Multiple organ failure & 5.719 & 1.774 to 18.436 & .004 \\
\hline $\begin{array}{l}\text { Serum creatinine } 24 \mathrm{~h} \text { after } \\
\text { surgery }\end{array}$ & 1.070 & 1.036 to 1.104 & .000 \\
\hline $\begin{array}{l}\text { Serum creatinine } 48 \mathrm{~h} \text { after } \\
\text { surgery }\end{array}$ & 1.071 & 1.039 to 1.103 & .000 \\
\hline Fresh-frozen plasma & 1.003 & 1.002 to 1.004 & .000 \\
\hline Packed red cells & 4.399 & 1.701 to 11.381 & .002 \\
\hline \multicolumn{4}{|l|}{ Multivariate analysis } \\
\hline Intubation time & 1.018 & 1.003 to 1.032 & .017 \\
\hline Chest drainage & 1.001 & 1.000 to 1.002 & .012 \\
\hline $\begin{array}{l}\text { Serum creatinine } 24 \mathrm{~h} \text { after } \\
\text { surgery }\end{array}$ & 1.057 & 1.011 to 1.105 & .014 \\
\hline $\begin{array}{l}\text { Serum creatinine } 48 \mathrm{~h} \text { after } \\
\text { surgery }\end{array}$ & 1.028 & 1.002 to 1.055 & .033 \\
\hline Fresh-frozen plasma & 1.002 & 1.000 to 1.003 & .037 \\
\hline Packed red cells & 9.536 & 1.493 to 60.930 & .017 \\
\hline
\end{tabular}

Abbreviation: ICU, intensive care unit.

the Medical Ethics Committee of the People's Hospital of Guangxi Zhuang Autonomous Region, which also gave the authors approval to waive the need for patient consent for publishing patient data from the study.

\section{RESULTS}

\section{Characteristics of the Population under Study}

92 consecutive patients undergoing pericardiectomy for constrictive pericarditis were included in the study. Only 2 patients underwent cardiac catheterization. No patient died within the first 24 hours after surgery. The patients were divided into 2 groups: with AKI $(\mathrm{n}=25)$ and without AKI $(\mathrm{n}=67)$ Table 1 shows the comparison of preoperative data between the patients with and without AKI.

\section{Operative Results}

Mortality and AKI. The incidence of postoperative AKI in the study was $27.2 \%$ (25/92). AKI occurred in 25 patients,

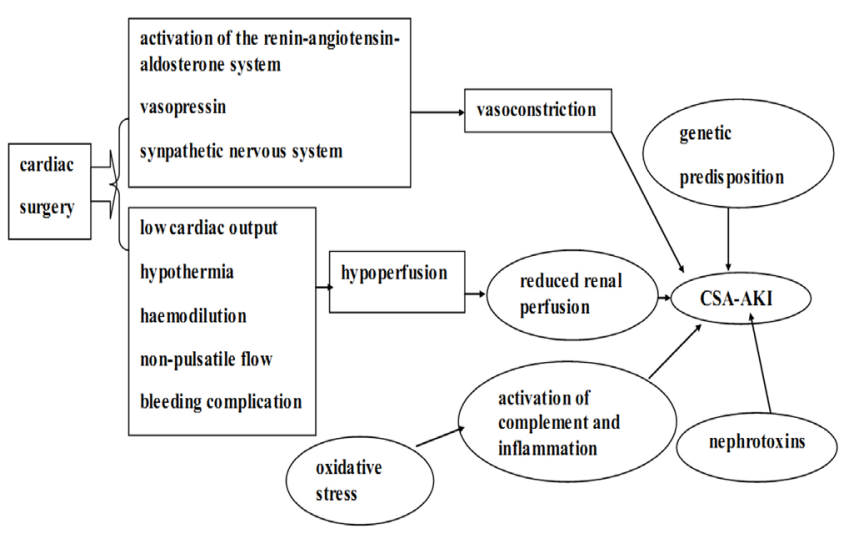

Figure 1. Pathophysiology of acute kidney injury after cardiac surgery.

of whom $11(44 \%)$ were classified as KDIGO stage 1, 4 $(16 \%)$ as KDIGO stage 2, and $10(40 \%)$ as KDIGO stage 3. Among the 25 patients who developed AKI, hemodialysis was required for $10(40 \%)$.

There were 5 operative deaths. Mortality in the group with AKI was significantly higher than in the group without AKI $(20 \%$ versus $0 \%, P=.000)$. Multiple organ failure in the group with AKI $(8 / 25,32 \%)$ was significantly higher than in the group without AKI $(0 / 25)$ (32\% versus $0 \%$, $P=.000)$.

Resource Utilization. Intubation time and time in ICU of the group with AKI were significantly longer than those of group without AKI $(133.7 \pm 23.5$ versus $54.3 \pm 6.5$ hours, $\mathrm{P}=.000 ; 7.6 \pm 1.3$ versus $4.5 \pm 0.5$ days, $P=.007$ ) (Table 2 ). Significantly more fresh-frozen plasma and packed red cells were used in the group with AKI than in the group without AKI $(1412.8 \pm 209.8$ versus $317.8 \pm 55.8 \mathrm{~mL}, P=.000 ; 0.58 \pm$ 0.2 versus $0.05 \pm 0.03$ units, $P=.000$ ) (Table 2 ).

Fluid Balance. Serum creatinine 24 and 48 hours after surgery increased significantly in the group with AKI. Fluid balance between the patients with and without AKI had significant differences (Table 2).

\section{Risk Factors of Acute Kidney Injury}

Table 3 shows the analysis of risk factors of acute kidney injury. In our study, both univariate and multivariate analyses showed that statistically significant independent predictors of acute kidney injury include intubation time, chest drainage, serum creatinine 24 and 48 hours after surgery, fresh-frozen plasma, and packed red cells (Table 3).

\section{Follow-Up Results}

All 87 survivors discharged from the hospital were monitored to the end date of the study, and the followup was $100 \%$ completed $(n=87)$. The mean duration of follow-up was $52.4 \pm 4.5$ months (range 2 to 138 ); no late deaths or reoperations occurred. The latest data of follow-up showed that 85 survivors were New York Heart Association (NYHA) class I (85/87, 97.7\%) and 2 in class II $(2 / 87,2.3 \%)$. 
Table 4. Risk Factors of the Development of Acute Kidney Injury after Cardiac Surgery

\begin{tabular}{lcc}
\hline Preoperative & Perioperative & Postoperative \\
\hline Advanced age & Complex surgery & Vasopressor exposure \\
Female gender & CPB duration & Inotrope exposure \\
Liver disease & Need to return to CPB & Diuretic exposure \\
Hyperlipidemia & Aortic cross-clamp time & Anemia \\
Previous stroke & Low hematocrit during & Blood transfusion \\
Chronic kidney disease & CPB & Hypovolemia \\
Hypertension & Emboli & Venous congestion \\
Smoking history & Venous congestion & Cardiogenic shock \\
$\begin{array}{l}\text { Peripheral vascular } \\
\text { disease }\end{array}$ & Hypoperfusion & \\
\hline
\end{tabular}

Abbreviations: AKI, acute renal injury; CPB, cardiopulmonary bypass.

\section{DISCUSSION}

\section{AKI Increases Mortality and Health Care Costs}

$\mathrm{AKI}$ is a condition characterized by an acute deterioration of renal function, as indicated by reduced glomerular filtration rate (GFR). Cardiac surgery-associated acute kidney injury, the second most common cause of AKI in the ICU, is a serious complication and the strongest predictor of mortality after surgery; it increases early and late mortality and health care costs [Hatton 2020; Fang 2020; Cho 2019; Engelman 2019; Rasmussen 2019]. In the present study, the incidence of postoperative AKI in the study was $27.2 \%$ (25/92). AKI occurred in 25 patients, of whom (\%) were classified as AKIN stage 1, whereas (\%) were classified as AKIN stage 2, and (\%) were classified as AKIN stage 3 . Among the 25 patients who developed AKI, hemodialysis was required for (\%). There were 5 operative deaths. Mortality and multiple organ failure in the group with AKI were significantly higher than in the group without $\mathrm{AKI}$ ( $40 \%$ versus $0 \%, P=.000$ ), and intubation time and time in ICU were significantly longer (Table 2). In our study, both univariate and multivariate analyses showed that statistically significant independent predictors of acute kidney injury include intubation time and serum creatinine 24 and 48 hours after surgery (Table 3 ).

\section{Pathophysiological Mechanisms of AKI}

The pathophysiology of AKI in the patients undergoing pericardiectomy involves multiple mechanisms: low cardiac output, inflammation, oxidative stress, nephrotoxins, metabolic abnormalities, and pre-existing chronic diseases. These factors also interact with one another pre-, peri-, and postoperatively. Figure 1 highlights the pathophysiological mechanisms resulting in development of AKI [Thomas 2020; Shen 2020; Nishimoto 2020; Sanaiha 2020; Murashima 2019; Ueno 2020].

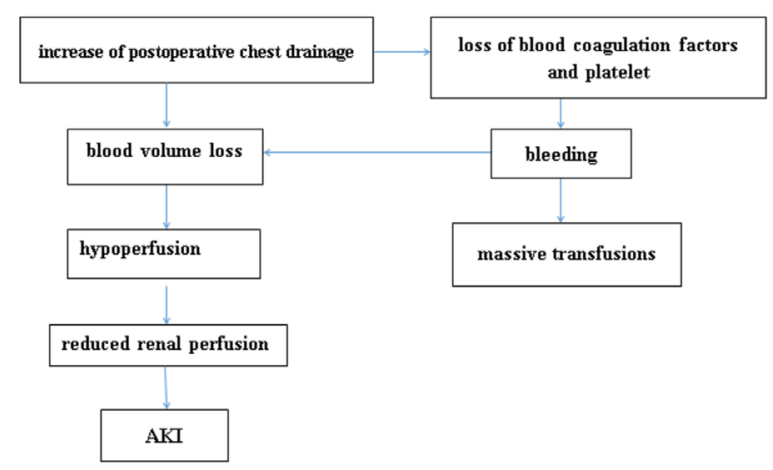

Figure 2. AKI group needed massive transfusions.

The early identification of patients at risk for developing AKI after cardiac surgery is an important strategy for improving the care of such patients. Epidemiological studies of AKI after cardiac surgery allow for better diagnosis of AKI and facilitate prognosis estimation and the development of new, more effective strategies to prevent and minimize this complication, thus reducing the associated morbidity and mortality [Padmanabhan 2019; Tseng 2020; Fang 2020].

The following factors have been found to facilitate the development of AKI after cardiac surgery: age, female sex, obesity, low cardiac output, blood transfusion, valve replacement surgery, heart failure, myocardial infarction in the last 30 days, chronic obstructive pulmonary disease, peripheral artery disease, prolonged $\mathrm{CPB}$, the use of inotropic or vasoconstrictor drugs, the use of an intra-aortic balloon pump, diabetes mellitus, systemic arterial hypertension, and chronic kidney disease [Dedemoğlu 2020; Husain-Syed 2020; Vlasov 2020; Hames 2019].

Studies have indicate that blood transfusion increases the likelihood of AKI occurring after cardiac surgery. In our study, significantly more fresh-frozen plasma and packed red cells were used in the group with AKI than in the group without AKI $(1412.8 \pm 209.8$ versus $317.8 \pm 55.8 \mathrm{~mL}, P=.000 ; 0.58 \pm$ 0.2 versus $0.05 \pm 0.03$ units, $P=.000$ ) (Table 2); Both univariate and multivariate analyses showed that chest drainage, freshfrozen plasma, and packed red cells were statistically significant independent predictors of acute kidney injury (Table 3.).

Studies have found an increased likelihood for females to develop AKI compared with males. Advancing age is associated with declining renal function and GFR, which compromises normal renal physiology and increases predisposition to developing AKI postoperatively. Patients with comorbidities such as diabetes mellitus, congestive cardiac failure, chronic obstructive pulmonary disease, and pre-existing chronic kidney disease are likely to be predisposed to developing renal insult after cardiac procedures. Patients with such comorbidities are frequently administered a variety of nephrotoxic medications, including angiotensin-converting enzyme inhibitors, nonsteroidal anti-inflammatory drugs, and angiotensin receptor blockers, all of which further adversely alter glomerular perfusion [Joannidis 2017; Yuan 2019; O’Neal 2019]. 
For KDIGO-AKI, higher body mass index, older age, female sex, chronic obstructive pulmonary disease, previous cardiac surgery, atrial fibrillation, NYHA class III or IV heart failure, higher preoperative serum creatinine, and the use of $\mathrm{CPB}$ were independent predictors of AKI after cardiac surgery [Kristovic 2015].

Preoperative cardiac insult is the risk factor associated with low cardiac output syndrome in the pre-, peri-, or postoperative period and increases the likelihood of developing postoperative AKI due to reduced perfusion pressures and global renal ischemic insult. Hemodynamic instability; nephrotoxic, vasoconstricting, and inotropic drugs; and systemic inflammation were the important factors influencing the development of AKI postoperatively. Moreover, pre-existing anemia or the development of anemia after surgery can also result in AKI owing to the reduced oxygen-carrying capacity of red blood cells and ischemic insult to the renal system. Table 4 is a summary of key factors contributing to the development of AKI after cardiac surgery [Romagnoli 2018; Wu 2019]. Figure 2 shows why the AKI group needed massive transfusions.

\section{Prevention of $A K I$}

Anemia and hyperglycemia are associated with elevated incidence of AKI, mortality, and morbidity. Thus it is important to avoid anemia, bleeding and hemolysis, and hyperglycemia. Goal-directed therapy is associated with a reduction in the incidence of AKI, renal replacement therapy, ICU stay, hospital stay, and decreased risk of renal dysfunction and reduced mortality [Ho 2015; Graziani 2019].

Perioperative fluid overload is also associated with increased severity of AKI and increased mortality after cardiac surgery. Positive fluid balance management is strongly associated with a higher AKI rate. Isotonic saline administration is associated with increased AKI risk due to excess chloride. Our study also showed that fluid balance between the patients with and without AKI had significant differences (Table 2).

\section{Management of $A K I$}

Prevention of AKI development after cardiac surgery is the optimal management, which includes optimization of multiple factors in the pre-, peri-, and postoperative periods, such as withholding nephrotoxic medications and preventing hypotension. Peri-operative measures should aim to improve renal reserve by improving perfusion pressures and, hence, reducing ischemic insult to the kidneys. Peri-operative hemofiltration can decrease systemic fluid overload and improve respiratory function in patients with background congestive heart failure [Ramos 2018].

Use of colloids is the best strategy to maintain intravascular volume and hemodynamic stability. Loop diuretics can be aggressively used if oliguric renal failure persists, which can often convert oliguric to non-oliguric renal failure, improving urine output, preventing tubular damage, and decreasing oxygen consumption. It is important to optimize hemodynamics, including reducing preload, increasing afterload, and improving the contractility of the heart to increase the cardiovascular efficacy, which prevents renal ischemia and ensures adequate renal perfusion to prevent renal tubular and parenchymal injuries [Park 2016]. Renal replacement is the only effective therapeutic intervention for patients with severe AKI or oliguric renal failure.

\section{Conclusions}

Acute kidney injury after pericardiectomy is a serious postoperative complication and contributes to a significant increase in perioperative morbidity and mortality rates. Prevention of AKI development after cardiac surgery and optimization of pre-, peri-, postoperative factors that can reduce AKI, therefore, contributes to a better postoperative outcome and leads to lower rates of AKI and morbidity and mortality.

\section{ACKNOWLEDGMENTS}

This work was supported by the Natural Science Foundation of China (grant 81360014), the Natural Science Foundation of Guangxi (grant 2014GXNSFAA118234), the Guangxi Key Scientific and Technological Project (grant 2013BC26236), the Projects in Guangxi Health Department (grant GZPT13-27), and the Projects in Guangxi Health Department (grant S2018075).

\section{REFERENCES}

Calderon-Rojas R, Greason KL, King KS, et al. Tricuspid valve regurgitation in patients undergoing pericardiectomy for constrictive pericarditis. Semin Thorac Cardiovasc Surg 2020;32:P721-P728.

Cho JS, Shim JK, Lee S, et al. Chronic progression of cardiac surgery associated acute kidney injury: Intermediary role of acute kidney disease. J Thorac Cardiovasc Surg 2019:161:681-688.e3.

Dedemoğlu M, Tüysüz ME. Risk estimation model for acute kidney injury defined by KDIGO classification after heart valve replacement surgery. Gen Thorac Cardiovasc Surg 2020;68:922-931.

Engelman DT, Crisafi C, Germain M, et al. Using urinary biomarkers to reduce acute kidney injury following cardiac surgery. J Thorac Cardiovasc Surg 2020:160:1235-1246.e2.

Fang L, Yu G, Huang J, Zhao W, Ye B. Predictors of postoperative complication and prolonged intensive care unit stay after complete pericardiectomy in tuberculous constrictive pericarditis. J Cardiothorac Surg 2020;15:148.

Graziani MP, Moser M, Bozzola CM, et al. Acute kidney injury in children after cardiac surgery: Risk factors and outcomes. A retrospective, cohort study. Arch Argent Pediatr 2019;117:e557-e567.

Hames DL, Ferguson MA, Kaza AK, et al. Renal replacement therapy in the pediatric cardiac intensive care unit. J Thorac Cardiovasc Surg 2019;158:1446-1455.

Hatton GE, Du RE, Wei S, et al. Positive fluid balance and association with post-traumatic acute kidney injury. J Am Coll Surg 2020;230:190199.e1.

Ho J, Tangri N, Komenda P, et al. Urinary, plasma, and serum biomarkers' utility for predicting acute kidney injury associated with cardiac surgery in adults: A meta-analysis. Am J Kidney Dis 2015;66:993-1005.

Howitt SH, Grant SW, Caiado C, et al. The KDIGO acute kidney injury guidelines for cardiac surgery patients in critical care: A validation study. 
BMC Nephrol 2018;19:149.

Husain-Syed F, Quattrone MG, Ferrari F, et al. Clinical and operative determinants of acute kidney injury after cardiac surgery. Cardiorenal Med 2020;10:340-352.

Jacob KA, Leaf DE. Prevention of cardiac surgery-associated acute kidney injury: A review of current strategies. Anesthesiol Clin 2019;37:729-749.

Joannidis M, Druml W, Forni LG, et al. Prevention of acute kidney injury and protection of renal function in the intensive care unit: update 2017: Expert opinion of the Working Group on Prevention, AKI section, European Society of Intensive Care Medicine. Intensive Care Med 2017;43:730-749.

Kristovic D, Horvatic I, Husedzinovic I, et al. Cardiac surgery-associated acute kidney injury: Risk factors analysis and comparison of prediction models. Interact Cardiovasc Thorac Surg 2015;21:366-373.

Leballo G, Chakane PM. Cardiac surgery-associated acute kidney injury: Pathophysiology and diagnostic modalities and management. Cardiovasc J Afr 2020;31:205-212.

Meersch M, Schmidt C, Hoffmeier A, et al. Prevention of cardiac surgery-associated AKI by implementing the KDIGO guidelines in high risk patients identified by biomarkers: the PrevAKI randomized controlled trial. Intensive Care Med 2017;43:1551-1561.

Murashima M, Nishimoto M, Kokubu M, et al. Inflammation as a predictor of acute kidney injury and mediator of higher mortality after acute kidney injury in non-cardiac surgery. Sci Rep 2019;9:20260.

Nishimoto M, Murashima M, Kokubu M, et al. Positive association between intra-operative fluid balance and post-operative acute kidney injury in non-cardiac surgery: The NARA-AKI cohort study. J Nephrol 2020;33:561-568.

O'Neal JB, Shaw AD, Billings FT 4th. Acute kidney injury following cardiac surgery: Current understanding and future directions. Crit Care 2016;20:187.

Padmanabhan H, Siau K, Curtis J, et al. Preoperative anemia and outcomes in cardiovascular surgery: Systematic review and meta-analysis. Ann Thorac Surg 2019;108:1840-1848.

Park SK, Hur M, Kim E, et al. Risk factors of acute kidney injury after congenital cardiac surgery in infants and children: A retrospective observational study. PLoS One 2016;11:e0166328.

Ramos KA, Dias CB. Acute kidney injury after cardiac surgery in patients without chronic kidney disease. Braz J Cardiovasc Surg 2018;33:454-461.

Rasmussen SR, Kandler K, Nielsen RV, et al. Duration of critically low oxygen delivery is associated with acute kidney injury after cardiac surgery. Acta Anaesthesiol Scand 2019;63:1290-1297.

Romagnoli S, Ricci Z, Ronco C. Perioperative acute kidney injury: Prevention, early recognition, and supportive measures. Nephron 2018;140:105-110.

Sanaiha Y, Kavianpour B, Dobaria V, et al. Acute kidney injury is independently associated with mortality and resource use after emergency general surgery operations. Surgery 2020;167:328-334.

Shen Z, Lin J, Teng J, et al. Association of urinary ionomic profiles and acute kidney injury and mortality in patients after cardiac surgery. J Thorac Cardiovasc Surg 2020;159:918-926.e5.

Sutherland L, Hittesdorf E, Yoh N, et al. Acute kidney injury after cardiac surgery: A comparison of different definitions. Nephrology (Carlton) 2020;25:212-218.

Thomas M, Kreibich M, Beyersdorf F, Benk C, Maier S, Trummer G. Standardized weaning from temporary extracorporeal life support in cardiovascular patients. Thorac Cardiovasc Surg 2020;68:425-432.

Tseng PY, Chen YT, Wang CH, et al. Prediction of the development of acute kidney injury following cardiac surgery by machine learning. Crit Care 2020;24:478.

Ueno K, Shiokawa N, Takahashi Y, et al. Kidney disease: Improving global outcomes in neonates with acute kidney injury after cardiac surgery. Clin Exp Nephrol 2020;24:167-173.

Vlasov H, Juvonen T, Hiippala S, et al. Effect and safety of $4 \%$ albumin in the treatment of cardiac surgery patients: study protocol for the randomized, double-blind, clinical ALBICS (ALBumin In Cardiac Surgery) trial. Trials 2020;21:235.

Wu B, Chen J, Yang Y. Biomarkers of acute kidney injury after cardiac surgery: A narrative review. Biomed Res Int 2019;2019:7298635.

Yuan SM. Acute kidney injury after cardiac surgery: Risk factors and novel biomarkers. Braz J Cardiovasc Surg 2019;34:352-360. 